\title{
Visual response shows promise as biomarker in autism-linked condition
}

\author{
BY LAURA DATTARO
}

23 AUGUST 2021

Brain responses to visual stimuli are smaller and weaker in children with Phelan-McDermid syndrome, an autism-linked genetic condition, than in non-autistic children, according to a new study. The difference in response is greater in children with larger genetic mutations.

Mutations or deletions in SHANK3, one of the genes most strongly linked to autism, cause PhelanMcDermid syndrome. More than 80 percent of people with the condition have autism; they also often have intellectual disability, developmental delays and other medical issues, though these traits and their severity can vary widely.

The new study is the first to use electroencephalography (EEG) to measure visual evoked potentials - brain responses that occur shortly after a person views a visual stimulus - in people with Phelan-McDermid syndrome. The team previously identified differences in these responses in people with 'idiopathic' autism, or autism with no known genetic cause. Other researchers have linked atypical visual evoked potentials to other single-gene causes of autism, such as Rett syndrome.

"Contributions like the one in this paper that allow us to have very clearly quantifiable biomarkers are extremely valuable," says Luigi Boccuto, assistant research scientist and head of the PhelanMcDermid syndrome research program at Clemson University in Clemson, South Carolina, who was not involved in the work. "They allow us to really categorize and better study and research the clinical features."

\section{Smaller responses:}

The researchers measured brain activity in 175 children aged 20 months to 12 years -31 with Phelan-McDermid and 20 of their unaffected siblings, and 79 with idiopathic autism and 45 without autism - while the children watched colors change on a checkerboard for 60 seconds. 


\section{Spectrum | Autism Research News}

https://www.spectrumnews.org

Children with Phelan-McDermid syndrome and idiopathic autism had similar responses, which were smaller than those of both the unaffected siblings and the non-autistic children.

A briefer version of the test, consisting of 10 two-second trials, produced similar results and allowed more children to participate.

"[This] offers flexibility when collecting objective EEG biomarker data in populations where such data has been historically difficult to obtain," says lead investigator Paige Siper, chief psychologist of the Seaver Autism Center at Mount Sinai Hospital in New York.

All of the children with Phelan-McDermid who have deletions in SHANK3, rather than mutations, also had greatly reduced brain responses, the researchers found; those with larger deletions had the most reduced responses, or no response. In contrast, some children with only point mutations changes to a single 'letter' of DNA - had more typical brain responses.

The results suggest that additional genes interact with SHANK3 and can influence how the condition presents, Boccuto says. "They may add more damage than the loss of SHANK3 alone. The breakthrough thing is that we can quantify that, so we can elaborate a better prognosis."

The findings were published in the Journal of the American Academy of Child and Adolescent Psychiatry in July.

\section{Clinical trials:}

Because the brain responses in children with different forms of autism overlapped, future therapies that are effective for Phelan-McDermid syndrome could potentially help other autistic children with similar neural patterns, Siper says.

And visual evoked potentials could be a useful tool for measuring the effects of future therapies, Boccuto says.

"Obviously the goal is to develop treatment that can fix these problems to some extent," he says. "So now you can measure the success of the treatment."

Biomarkers have been particularly difficult to identify in Phelan-McDermid syndrome, says Jimmy Lloyd Holder Jr, assistant professor of pediatrics, neurology and developmental neuroscience at Baylor College of Medicine in Houston, Texas, who was not involved in the work. This is in part because many people with the condition also have intellectual disability, which can make it difficult for them to participate in some data-gathering processes, such as functional magnetic resonance imaging.

"Really, prior to this, there's been very little for Phelan-McDermid syndrome," Holder says. 


\section{Spectrum | Autism Research News}

https://www.spectrumnews.org

EEG is easier to conduct and doesn't require the sedation often used with brain imaging, Holder says, making this a promising technique. Many children with Phelan-McDermid already have EEGs done to monitor seizure activity.

Future work should include a control group with idiopathic intellectual disability, Holder says, and should investigate whether evoked potentials are associated with specific behavioral traits.

Siper's team is evaluating the use of visual evoked potentials in clinical trials involving children with Phelan-McDermid and examining whether they are an effective biomarker in other autismassociated genetic conditions.

Cite this article: https://doi.org/10.53053/VXPR9239 